\title{
Intratracheal Cell Transfer Demonstrates the Profibrotic Potential of Resident Fibroblasts in Pulmonary Fibrosis
}

\author{
Tatsuya Tsukui, ${ }^{* \dagger}$ Satoshi Ueha, ${ }^{* \dagger}$ Shigeyuki Shichino, ${ }^{* \dagger}$ Yutaka Inagaki, ${ }^{\dagger \dagger}$ and Kouji Matsushima* ${ }^{* \dagger}$
}

\begin{abstract}
From the Department of Molecular Preventive Medicine,* Graduate School of Medicine, The University of Tokyo, Tokyo; the Japan Agency for Medical Research and Development-Core Research for Evolutional Science and Technology (AMED-CREST) Program, ${ }^{\dagger}$ Tokyo; and the Center for Matrix Biology and Medicine, ${ }^{\ddagger}$ Graduate School of Medicine, Tokai University, Kanagawa, Japan
\end{abstract}

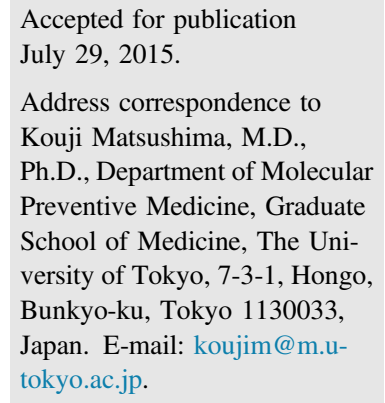

\begin{abstract}
Pulmonary fibrosis is a devastating disease for which there are few effective therapies. Activated fibroblasts form subepithelial clusters known as fibroblastic foci, which are characterized by excessive collagen deposition. The origin of activated fibroblasts is controversial and needs to be clarified to understand their pathogenicity. Here, using an intratracheal adoptive cell transfer method, we show that resident fibroblasts in alveolar walls have the highest profibrotic potential. By using collagen $\mathrm{I}(\alpha) 2$-green fluorescent protein and neural/glial antigen 2-DsRed fluorescent reporter mice, we identified resident fibroblasts and pericytes in the alveolar walls based on surface marker expression and ultrastructural characteristics. In the early phase of bleomycin-induced pulmonary fibrosis, activated fibroblasts migrated into epithelium-denuded alveolar airspaces. Purified resident fibroblasts delivered into injured alveoli by an intratracheal route showed similar activated signatures as activated fibroblasts and formed fibroblastic foci. Neither pericytes nor epithelial cells had the same profibrotic potential. Transferred resident fibroblasts highly up-regulated profibrotic genes including $\alpha$-smooth muscle actin and were a significant source of collagen deposition. These data provide insights into the cellular mechanisms of fibrogenesis and show intratracheal cell transfer to be a useful tool for exploring novel therapeutic targets against pulmonary fibrosis. (Am J Pathol 2015, 185: 2939-2948; http://dx.doi.org/10.1016/j.ajpath.2015.07.022)
\end{abstract}

Idiopathic pulmonary fibrosis (IPF) is a progressive interstitial lung disease of unknown etiology that is characterized by architectural destruction of alveoli and the formation of fibroblastic foci. ${ }^{1-3}$ Activated fibroblasts, such as myofibroblasts that express $\alpha$-smooth muscle actin ( $\alpha$-SMA), accumulate in fibroblastic foci and deposit excessive amounts of extracellular matrix components such as type 1 collagen, leading to loss of organ function. Characterization of the pathogenic effects of activated fibroblasts will help to clarify the underlying mechanisms in fibrotic diseases and support the development of specific treatment strategies against fibrosis.

The origin of activated fibroblasts in fibrotic diseases remains controversial. ${ }^{4}$ Although previous reports have proposed a contribution of circulating fibrocytes or epithelial cells through epithelial-to-mesenchymal transition, recent studies using parabiosis or epithelial cell-specific Cre-reporter mice in a model of bleomycin-induced pulmonary fibrosis have challenged these views. ${ }^{5,6}$ A recent lineage tracing study showed a major contribution of Foxd1-Cre-labeled lung pericytes to the myofibroblast population. ${ }^{7}$ There is mounting evidence that perivascular cells including pericytes play significant roles in fibrosis in multiple organs. ${ }^{8-10}$ However, it is not clear whether the cell populations described in these studies play equivalent roles in all organs and diseases. Moreover, there is a lack of studies that have performed rigorous lineage tracing of resident fibroblasts, which classically have been suggested to be major progenitors of myofibroblasts. In the present study, we performed lineage tracing of

Supported by AMED, CREST, a Japan Society for the Promotion of Science fellowship (DC1 to T.T.), and by The University of Tokyo Life Innovation Leading Graduate School, Graduate Program for Leaders in Life Innovation (T.T. and S.S.).

Disclosures: None declared. 
resident fibroblasts using intratracheal adoptive cell transfer. By this approach, we show that resident fibroblasts have the highest profibrotic potential compared with other lung cell populations. Transferred resident fibroblasts formed fibroblastic foci similar to those observed in IPF patients, suggesting a profibrotic role of resident fibroblasts in pulmonary fibrosis.

\section{Materials and Methods}

Mice

Collagen I $(\alpha)$ 2-green fluorescent protein (GFP) reporter mice (Col-GFP mice; C57BL/6 background) were generated in a previous study. ${ }^{11}$ Neural/Glial antigen 2 (NG2)-DsRed mice were purchased from the Jackson Laboratory (stock number 008241; Bar Harbor, ME). NG2-DsRed mice were crossed with either Col-GFP or C57BL/6 mice and the F1 offspring were used for experiments after genotyping. ROSA-CAGSCAT3.1 mice were generated from BDF1-derived embryonic stem cells and back-crossed to C57BL/6 mice for at least two generations. ${ }^{6,12}$ Host C57BL/6 mice used for intratracheal transfer experiments were purchased from CLEA Japan (Tokyo, Japan). Experiments were performed on 8- to 12 -week-old mice. Mice were maintained in specific pathogen-free facilities at the University of Tokyo. All animal experiments were performed in accordance with the guidelines of the Animal Care and Use Committee of the University of Tokyo.

\section{Intratracheal Instillation and BrdU Treatment}

Intratracheal instillation for bleomycin (BLM) treatment or cell transfer experiments was performed as described previously. ${ }^{6}$ Briefly, mice were anesthetized before instillation by oropharyngeal aspiration with $50 \mu \mathrm{L}$ of either 2.0 to $2.5 \mathrm{mg} / \mathrm{kg}$ BLM sulfate (Toronto Research Chemical, Toronto, Canada) or saline as a negative control. For intratracheal transfer experiments, $50 \mu \mathrm{L}$ of either cell suspension or phosphatebuffered saline (PBS) vehicle were instilled in the same way. For bromodeoxyuridine (BrdU) incorporation analysis, 0.8 $\mathrm{mg} / \mathrm{mL}$ BrdU (Sigma-Aldrich, St. Louis, MO) was included in the drinking water of host mice commencing 1 day before intratracheal transfer and continuing until the day of analysis.

\section{Tissue Dissociation}

Lung cell suspensions were prepared as described previously, with minor modifications. ${ }^{6}$ Briefly, lungs were harvested after perfusion with $5 \mathrm{~mL}$ of PBS via the right ventricle. The lobes were minced and digested in protease solution $[0.2 \%$ collagenase (Wako Pure Chemical Industries, Osaka, Japan), $0.1 \mathrm{mg} / \mathrm{mL}$ Dispase II (Roche, Basel, Switzerland), and $2000 \mathrm{U} / \mathrm{mL}$ DNase I (Merck, Darmstadt, Germany) in RPMI medium (Sigma-Aldrich) supplemented with $10 \mathrm{mmol} / \mathrm{L}$ HEPES (Nacalai Tesque,
Kyoto, Japan)] for 60 minutes at $37^{\circ} \mathrm{C}$ with trituration by micropipette every 20 minutes. After digestion, cells were passed through a 70- $\mu \mathrm{m}$ strainer (BD Biosciences, San Jose, CA), washed with PBS, and suspended in RPMI medium with $0.5 \%$ bovine serum albumin (Nacalai Tesque).

\section{Antibodies}

The antibodies used were as follows: anti-CD31 (clone 390; BV421, PerCP/Cy5.5, and biotin; BioLegend, San Diego, CA), anti-CD31 (AF3628; R\&D Systems, Minneapolis, $\mathrm{MN}$ ), anti-CD45 [clone 30-F11; allophycocyanin (APC)/ Cy7 and biotin; BD Biosciences], anti-CD45.2 (clone 104; APC; BioLegend), anti-platelet-derived growth factor receptor (PDGFR) $\alpha$ (clone APA5; APC and biotin; BioLegend), anti-PDGFR $\beta$ (AF1042; R\&D Systems), antiPDGFR $\beta$ (clone C82A3; Cell Signaling Technology, Danvers, MA), anti-CD146 (clone ME-9F1; PerCP/Cy5.5, APC, and unlabeled; BioLegend), anti-epithelial cell adhesion molecule (EpCAM) [clone G8.8; phycoerythrin (PE)/Cy7, APC/Cy7, and biotin; BioLegend], anti-Ter119 (clone Ter119; APC/Cy7 and biotin; BD Biosciences), anti- $\alpha-$ SMA (clone 1A4; APC; R\&D Systems), anti-collagen I (LSL-LB-1102; LSL, Tokyo, Japan), anti-collagen IV (LSL-LB-1403; LSL), anti-BrdU (clone Bu20a; APC; BioLegend), anti-BrdU (clone MoBu-1; Alexa 647; Life Technologies, Grand Island, NY), anti-APC (130-090-855; microbeads; Miltenyi Biotec, San Diego, CA), anti-goat IgG (A-21447; Alexa 647; Life Technologies), anti-rabbit IgG (A-31572 and A-31573; Alexa 555 and 647; Life Technologies), and anti-rat IgG (A-21247; Alexa 647; Life Technologies).

\section{Flow Cytometry}

Cell numbers in whole-lung cell suspensions were counted using Flow-Count Fluorospheres (Beckman Coulter, Brea, CA). Subsequently, cells were incubated with an anti-CD16/32 antibody to block nonspecific binding, then stained for lineage markers. For $\alpha$-SMA staining, cells were fixed with Cytofix/ Cytoperm buffer (BD Biosciences) for 20 minutes before staining with the anti- $\alpha$-SMA antibody. BrdU incorporation was analyzed using a BrdU flow kit (BD Biosciences). Antibody-labeled cells were washed with PBS and analyzed using a Gallios flow cytometer (Beckman Coulter). Flow cytometry data were analyzed using FlowJo software version 7.6.5 (Tree Star, Ashland, OR).

\section{Cell Purification}

For enrichment of Col-GFP ${ }^{+} \mathrm{PDGFR}^{+}$resident fibroblasts, whole-lung cell suspensions were stained for CD31, CD45, EpCAM, and Ter119, followed by negative selection using an AutoMACS cell separator (Miltenyi Biotec). Cells in negative fractions were checked for purity and cell number. After calculating the cell concentration, cells were washed and suspended in PBS for intratracheal transfer. For 
fluorescence-activated cell sorting purification of Col-GFP ${ }^{+}$, Col-GFP $^{+}$NG2-DsRed ${ }^{+}$, NG2-DsRed ${ }^{+}$, and EpCAM $^{+}$ cells, whole-lung cell suspensions were divided into two fractions. One fraction was stained for CD31, CD45, CD146, and Ter119 before negative selection by AutoMACS, after which the negative fractions were stained for EpCAM and then sorted with a MoFlo Astrios flow cytometer (Beckman Coulter) for Col-GFP ${ }^{+}$autofluorescence ${ }^{+}$cells or $\mathrm{EpCAM}^{+}$ cells. The other fraction was stained for CD31, CD45, PDGFR $\alpha$, EpCAM, and Ter119 before negative selection by AutoMACS, after which the negative fractions were sorted with the MoFlo Astrios flow cytometer for Col-GFP ${ }^{+}$NG2DsRed $^{+}$cells or NG2-DsRed ${ }^{+}$cells. Sorted cells were checked for purity and cell number, and suspended in PBS ready for intratracheal transfer.

\section{Immunohistochemistry}

Immunohistochemistry was performed as described previously. ${ }^{6} \mathrm{Z}$-stack images were reconstructed in 3D by surface rendering using Velocity 3D image analysis software version 6.2.1 (PerkinElmer, Waltham, MA).

\section{Quantitative Real-Time PCR Analysis}

Col-GFP ${ }^{+}$cells $\left(1 \times 10^{7}\right)$ were transferred into host mice on day 7 after BLM treatment. At an additional 7 days after intratracheal transfer, whole lungs were harvested and wholelung cell suspensions were stained for CD31, CD45, CD146, EpCAM, and Ter119 before negative selection by AutoMACS. Whole-lung cells also were prepared from untreated Col-GFP mice as a negative control. Col-GFP ${ }^{+}$cells were sorted from the AutoMACS negative fraction using a MoFlo Astrios flow cytometer. After confirming cell purity, cells were lyzed in TRIzol reagent (Life Technologies) and total RNA was isolated according to the manufacturer's protocol. RNA was reverse-transcribed to cDNA using a ReverTra Ace qPCR RT Kit (Toyobo, Osaka, Japan). Quantitative real-time PCR was performed using Thunderbird SYBR qPCR Mix (Toyobo) with an ABI 7500 real-time PCR system (Life Technologies). The primers used were as follows: Rps3 forward $5^{\prime}-\mathrm{CG}$ GTGCAGATTTCCAAGAAG- $3^{\prime}$ and reverse $5^{\prime}$-GGACTTCAACTCCAGAGTAGCC-3'; Collal forward 5'-AGACATGTTCAGCTTTGTGGAC- $3^{\prime}$ and reverse $5^{\prime}$-GCAGCTGACTTCAGGGATG-3'; Acta2 forward 5'-GTCCCAGACATCAGGGAGTAA- $3^{\prime}$ and reverse 5'-TCGGATACTTCAGCGTCAGGA-3'; Spp1 forward 5'-GGAGGAAACCAGCCAAGG-3' and reverse $5^{\prime}$-TGCCAGAATCAGTCACTTTCAC-3'; S100a4 forward 5'-GGAGCTGCCTAGCTTCCTG- $3^{\prime}$ and reverse $5^{\prime}$-TCCTGGAAGTCAACTTCATTGTC-3'; Fn 1 forward $5^{\prime}$-CGGAGAGAGTGCCCCTACTA-3' and reverse $5^{\prime}$-CGATATTGGTGAATCGCAGA-3'; Tnc forward $5^{\prime}$-GGGCTATAGAACACCGATGC- $3^{\prime}$ and reverse 5'-CATTTAAGTTTCCAATTTCAGGTTC- ${ }^{\prime}$; Il1b forward 5'-AGGCAGGCAGTATCACTCATTGT-3' and reverse 5'-CGTCACACACCAGCAGGTTATC-3'; II5 forward
5'-AAGGATGCTTCTGCACTTGAGT- $3^{\prime}$ and reverse 5'-TCTCCAATGCATAGCTGGTGAT-3'; 1113 forward 5'-CTTGCCTTGGTGGTCTCG-3' and reverse 5'-CGTTGCACAGGGGAGTCT-3'; Il17a forward 5'-AAGCTCAGCGTGTCCAAACA-3' and reverse 5'-GGCACTGAGCTTCCCAGATC-3'; and Tgfb1 forward 5'-CCCGAAGCGGACTACTATGC- $3^{\prime}$ and reverse 5'-CCCGAATGTCTGACGTATTGAA-3'. Relative gene expression levels were calculated after normalization to expression of the reference gene Rps3.

\section{Migration Assay}

The migratory capacity of Col-GFP ${ }^{+}$cells was evaluated using an Oris cell migration assay kit (fibronectin-coated) (Platypus Technologies, Madison, WI). After purifying Col-GFP $^{+}$cells, $1 \times 10^{5}$ cells in $100 \mu \mathrm{L}$ of Dulbecco's modified Eagle's medium (DMEM) medium (SigmaAldrich) containing 2\% fetal bovine serum (Sigma-Aldrich) were dispensed into each well of a 96-well plate containing a cell-seeding stopper. After a 24-hour incubation in a $\mathrm{CO}_{2}$ incubator, the stopper was removed and the wells were washed with PBS to remove nonadherent cells. The wells were refilled with $100 \mu \mathrm{L}$ DMEM containing $2 \%$ fetal bovine serum; images of the Col-GFP ${ }^{+}$cells were acquired using an SP-5 confocal microscope (Leica Microsystems, Wetzlar, Germany) after a further 0,12 , or 24 hours of incubation. The area of the cell-free zone on the center of each well was quantified using ImageJ version $1.47 \mathrm{t}(\mathrm{NIH}$, Bethesda, MD; http://imagej.nih.gov/ij). The migration index was calculated as a ratio relative to the cell-free area at time 0 hour.

\section{Invasion Assay}

Invasion assays were performed using the Cultrex 96-well basement membrane extract cell invasion assay kit (Trevigen, Gaithersburg, MD). Purified Col-GFP ${ }^{+}$cells on plastic dishes were incubated in DMEM containing $2 \%$ fetal bovine serum for 24 hours. After trypsinizing the cells, $5 \times 10^{4}$ cells in serumfree DMEM were dispensed into the upper chambers of the assay kit, which were coated with $0.5 \times$ basement membrane extract. The lower chambers were filled with DMEM containing $2 \%$ fetal bovine serum. After a 36-hour incubation, all cells were fixed with $4 \%$ paraformaldehyde (Nacalai Tesque), and the cells on the upper side of the membrane were removed by wiping with a cotton swab. The cells on the lower side of the membrane were stained with Diff-Quik (Sysmex, Kobe, Japan) and counted. The invasion index was calculated by normalizing the cell number for treated Col-GFP ${ }^{+}$cells to the average cell number for control Col-GFP ${ }^{+}$cells.

\section{Hydroxyproline Assay}

Hydroxyproline assays were performed as described previously. ${ }^{6}$ 


\section{Statistical Analysis}

Data are expressed as means \pm SEM where applicable. Statistical comparisons were performed by unpaired $t$-tests (two-tailed) or by one-way analysis of variance with the Tukey-Kramer post-test for multiple groups. $P<0.05$ was considered statistically significant. Statistical analysis was performed using Prism software version 5.01 (GraphPad Software, La Jolla, CA).

\section{Results}

The lungs are composed of numerous types of cells that contribute to the complex structure of the organ and enable efficient gas exchange. ${ }^{13}$ A clear classification of stromal cell subsets in the healthy state is necessary to understand cellular origin in pathologic conditions. We previously showed that resident fibroblasts in the alveolar walls are labeled with GFP in Col-GFP mice. ${ }^{6}$ To further characterize the resident fibroblast population and to determine the overlap between the Col-GFP ${ }^{+}$population and other stromal cell populations, we cross-bred Col-GFP mice with NG2-DsRed mice. NG2 is expressed on some, but not all, pericytes. $^{7,14}$ Flow cytometric analysis of whole-lung cells from Col-GFP ${ }^{+/-}$NG2-DsRed ${ }^{+/-}$mice showed three distinct transgene-expressing populations in lineage marker-negative (ie, CD45- Ter119- EpCAM $^{-} \mathrm{CD}^{-} 1^{-}$) cells: $\mathrm{Col}_{-\mathrm{GFP}^{+}}$(population R1), Col-GFP ${ }^{+} \mathrm{NG}^{-\mathrm{DsRed}^{+}}$(population R2), and NG2-DsRed ${ }^{+}$(population R3) (Figure 1A). It has been reported previously that PDGFR $\alpha$ is expressed on resident fibroblasts and that CD146 is expressed on smooth muscle cells or pericytes. ${ }^{6,7,15}$ The $\mathrm{R} 1$ population contained $86.7 \% \pm 1.29 \%$ $\mathrm{PDGFR}^{+}$cells and $5.26 \% \pm 0.37 \% \mathrm{CD}^{4} 46^{+}$cells (Figure 1A), whereas the R2 and R3 populations were composed mostly of CD146 ${ }^{+}$cells (Figure 1A).

Histologically, peribronchiolar and perivascular Col-GFP ${ }^{+}$ cells were $\mathrm{CD}_{146^{+}}$(Figure 1B). Considering that perivascular but not peribronchiolar smooth muscle cells had NG2-DsRed expression (Figure 1B), CD146 ${ }^{+}$cells in the R1 population are likely to represent peribronchiolar smooth muscle cells, whereas the $\mathrm{CD} 146^{+}$cells in the $\mathrm{R} 2$ population are likely to represent perivascular smooth muscle cells. In the alveolar area, we observed NG2-DsRed ${ }^{+}$cells, which also expressed PDGFR $\beta$ (a marker of pericytes), but not all PDGFR $\beta^{+}$cells expressed NG2-DsRed (Figure 1C). In addition, we occasionally detected complete overlap of NG2-DsRed signals with Col-GFP signals in the alveolar walls $(8.5 \% \pm 1.4 \%$ of NG2-DsRed ${ }^{+}$cells) (Supplemental Figure S1A). Although some of these NG2-DsRed ${ }^{+}$Col-GFP ${ }^{+}$regions could represent the overlap of two distinct cells, it also is possible that some pericytes have a fibroblast-like phenotype, as reported previously. ${ }^{7}$ Ultrastructural analysis of NG2-DsRed ${ }^{+}$cells in the alveolar walls confirmed characteristics typical of pericytes, which were located in close proximity to CD $31^{+}$ endothelial cells and were enveloped together by the endothelial basement membrane (Figure 1D and Supplemental Movie S1). ${ }^{16}$ Thus, the R3 population represented a proportion of the pericytes in the alveolar walls, whereas the R2 population represented a mix of perivascular smooth muscle cells and, to a lesser extent, Col-GFP ${ }^{+}$DsRed $^{+}$cells in the alveolar walls. Col-GFP ${ }^{+}$cells in alveolar walls expressed PDGFR $\alpha$ (Figure 1E), and Col-GFP ${ }^{+}$PDGFR $\alpha^{+}$cells showed autofluorescence when excited by a violet laser, possibly owing to their storage of lipid droplets (Supplemental Figure S1B). ${ }^{17,18}$ An endothelial basement membrane separated Col-GFP ${ }^{+}$cells from the majority of the endothelium, a characteristic that distinguished these cells from PDGFR $\beta^{+}$pericytes, which were convoluted within the endothelial basement membrane (Figure 1, F and G). These features of Col-GFP ${ }^{+}$cells were consistent with the ultrastructural definition of resident fibroblasts in the alveolar walls. ${ }^{19}$

Col-GFP $^{+}$cells form clusters after BLM-induced injury, whereas they exist only sparsely in alveolar walls in the normal state. ${ }^{6}$ Col-GFP ${ }^{+}$cells in the alveolar walls were surrounded by a basement membrane and epithelial cells in the normal state (Figure 2A). In the early phase of BLM-induced injury, some $\mathrm{Col}-\mathrm{GFP}^{+}$cells migrated out of the interstitium into alveolar airspaces, with one end of the cell remaining attached to the denuded alveolar walls (Figure 2B). Consistent with this observation, Col-GFP ${ }^{+}$cells isolated at day 7 after bleomycin treatment showed increased migratory capacity as assessed by a gap migration assay and increased invasive capacity (Supplemental Figure S2, A-C). The migration of fibroblasts into alveolar airspaces also has been described in electron microscopy studies of experimental lung fibrosis or IPF. ${ }^{20,21}$ In those studies, the investigators reported that fibroblasts with well-developed endoplasmic reticula are exposed to alveolar airspaces after epithelial injury, and that these fibroblasts attach to the luminal surface of epithelial basement membranes. ${ }^{20}$ Fibroblastic foci in IPF patients are considered to be formed by the exudation of fibroblasts into alveolar airspaces. $^{22}$ To investigate whether fibroblasts in alveolar airspaces develop into fibroblast clusters in the interstitium, we isolated resident fibroblasts from the lungs of untreated Col-GFP mice and transferred this cell suspension into BLMtreated, wild-type mice via an intratracheal route (intratracheal

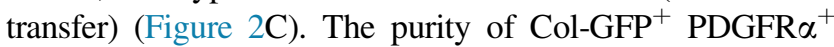
cells was greater than $80 \%$ in each experiment (Supplemental Figure S2D). On day 21 after BLM treatment, we detected Col-GFP ${ }^{+}$cells by both flow cytometry and immunohistochemistry (Figure 2, D and E, and Supplemental Figure S2E). In contrast, Col-GFP ${ }^{+}$cells were undetectable when transferred into saline-treated mice (Figure 2, D and E). We observed more Col-GFP ${ }^{+}$cells in the groups transferred on days 7 or 10 after BLM treatment compared with those transferred on day 4 after BLM treatment (Figure 2D), probably reflecting the increased epithelial injury present at the later time points. ${ }^{6}$

One day after intratracheal transfer (dpit), most Col-GFP ${ }^{+}$ cells had a round shape and remained in the alveolar airspaces (Figure 2F), where they attached to alveolar epithelial surfaces 


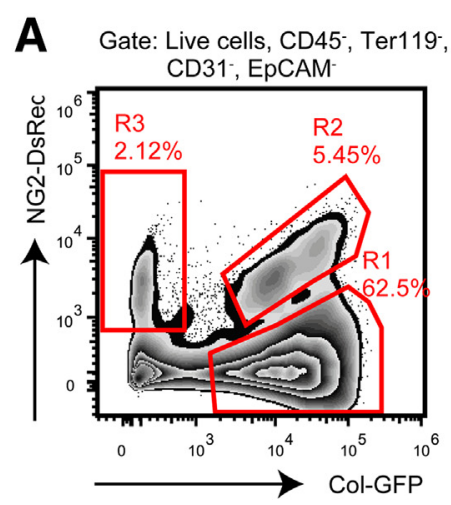

R1

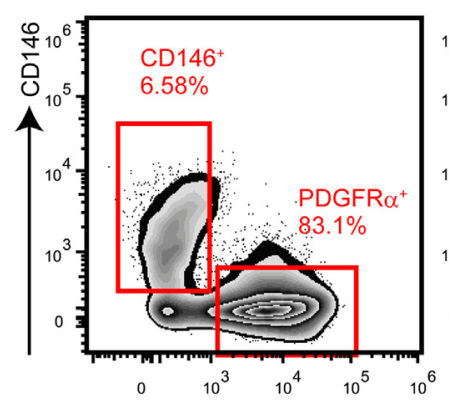

R2

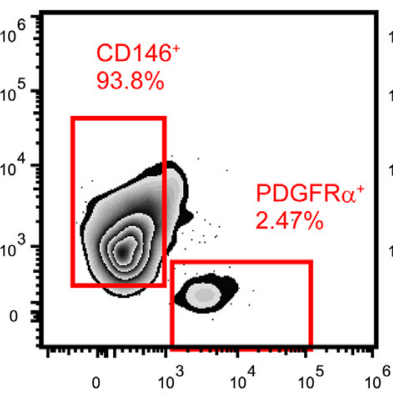

R3

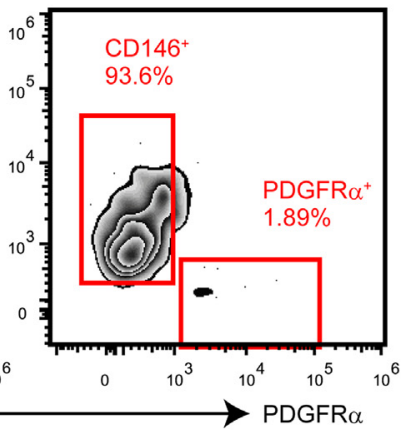

B

Col-GFP NG2-DsRed
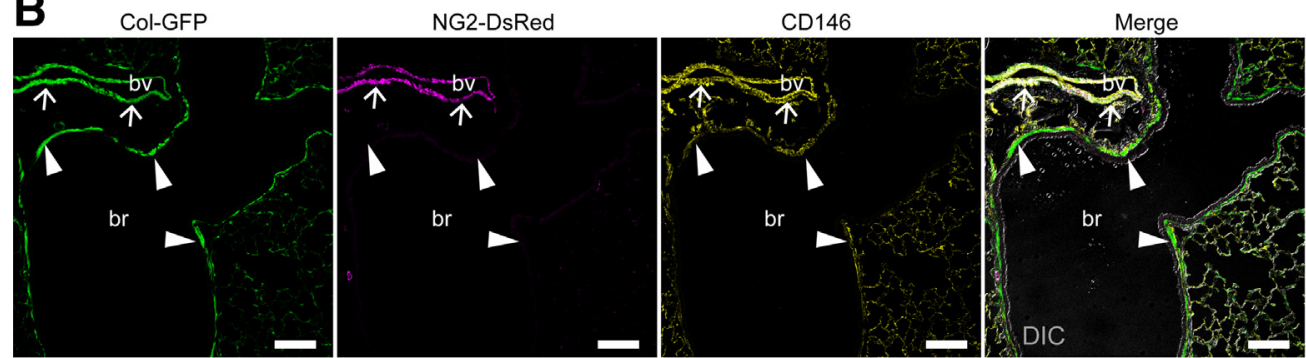

C NG2-DsRed
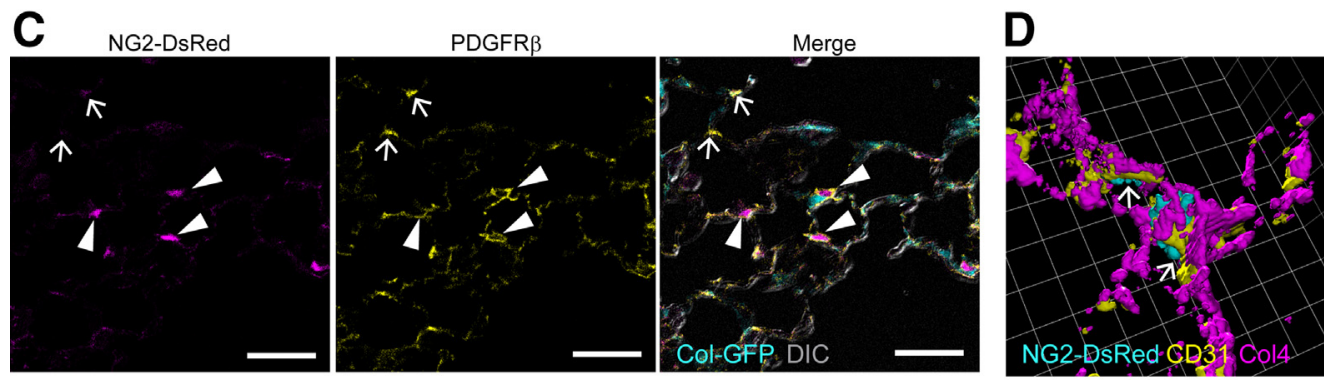

E Col-GFP
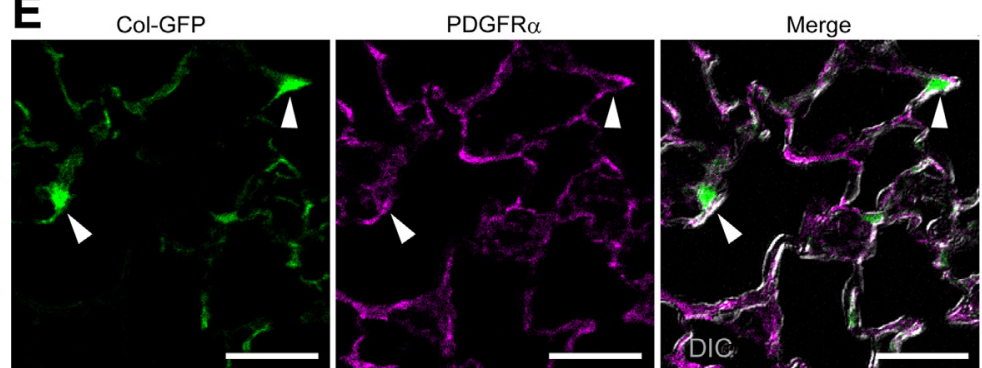

$\mathbf{F}$

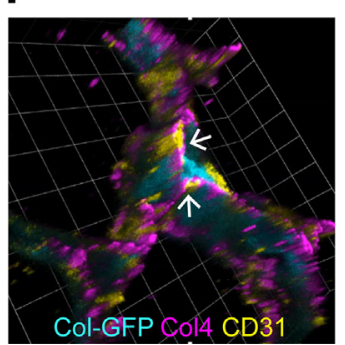

G

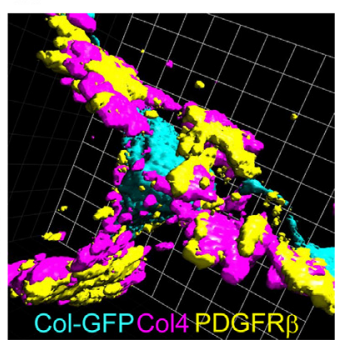

Figure 1 Analysis of stromal cell heterogeneity in Col-green fluorescent protein (GFP) NG2-DsRed mice. A: Whole-lung cells were analyzed by flow cytometry. Right panels show the R1, R2, and R3 populations gated in the left panel. B: Lung sections from Col-GFP (green) NG2-DsRed (magenta) mice were stained for CD146 (yellow). Arrowheads indicate CD146 ${ }^{+}$peribronchiolar smooth muscle cells. Arrows indicate NG2-DsRed ${ }^{+}$CD146 $^{+}$perivascular smooth muscle cells. C: Lung sections from Col-GFP (cyan) NG2-DsRed (magenta) mice were stained for platelet-derived growth factor receptor (PDGFR) $\beta$ (yellow). Arrowheads indicate PDGFR $\beta^{+}$NG2-DsRed ${ }^{+}$cells. Arrows indicate PDGFR $\beta^{+}$NG2-DsRed cells. D: Lung sections from NG2-DsRed (cyan) mice were stained for CD31 (yellow) and type 4 collagen (Col4, magenta). A series of Z-stack images were reconstructed in 3D. Arrows: NG2-DsRed ${ }^{+}$pericytes in close proximity to $\mathrm{CD} 1^{+}$cells, both of which were enveloped by the endothelial basement membrane. E: Lung sections from Col-GFP (green) mice were stained for PDGFR $\alpha$ (magenta). Arrowheads indicate Col-GFP ${ }^{+}$PDGFR $\alpha^{+}$cells. $\mathbf{F}$ and G: Lung sections from Col-GFP (cyan) mice were stained for Col4 (magenta), CD31 (F, yellow), and PDGFR $\beta$ (G, yellow). A series of Z-stack images were reconstructed in 3D. Arrows indicate separation of the majority of CD31 ${ }^{+}$cells from Col-GFP ${ }^{+}$cells by Col4. At least three independent experiments were performed for each analysis. Scale bars: $100 \mu \mathrm{m}$ (B), $50 \mu \mathrm{m}$ (C), $30 \mu \mathrm{m}$ (E). br, bronchiole; bv, blood vessel; DIC, differential interference contrast.

or the denuded epithelial basement membrane. By 4 dpit, Col$\mathrm{GFP}^{+}$cells had become dilated and changed their morphology dramatically (Figure 2F). These enlarged Col-GFP ${ }^{+}$cells increased the surface area attached to the reticular network of the basement membrane. At 7 dpit, Col-GFP ${ }^{+}$cells with a stellate shape appeared to be incorporated completely into the interstitium and some of them formed fibroblastic foci similar to those seen in BLM-treated Col-GFP mice (Figure 2, E and F). ${ }^{6}$ These results indicate that resident fibroblasts delivered into injured alveoli were activated by 
the inflammatory environment and incorporated into fibrotic lesions. Taken together with the suggested role of migratory fibroblasts in generating fibroblastic foci, ${ }^{23}$ intratracheal transfer partly recapitulates the process of fibroblast migration and cluster formation, and shows that some fibroblasts in fibroblastic foci originate from the alveolar airspaces.

Because we found that Col-GFP ${ }^{+}$cells up-regulate profibrotic genes after BLM-induced injury in our previous study, ${ }^{6}$
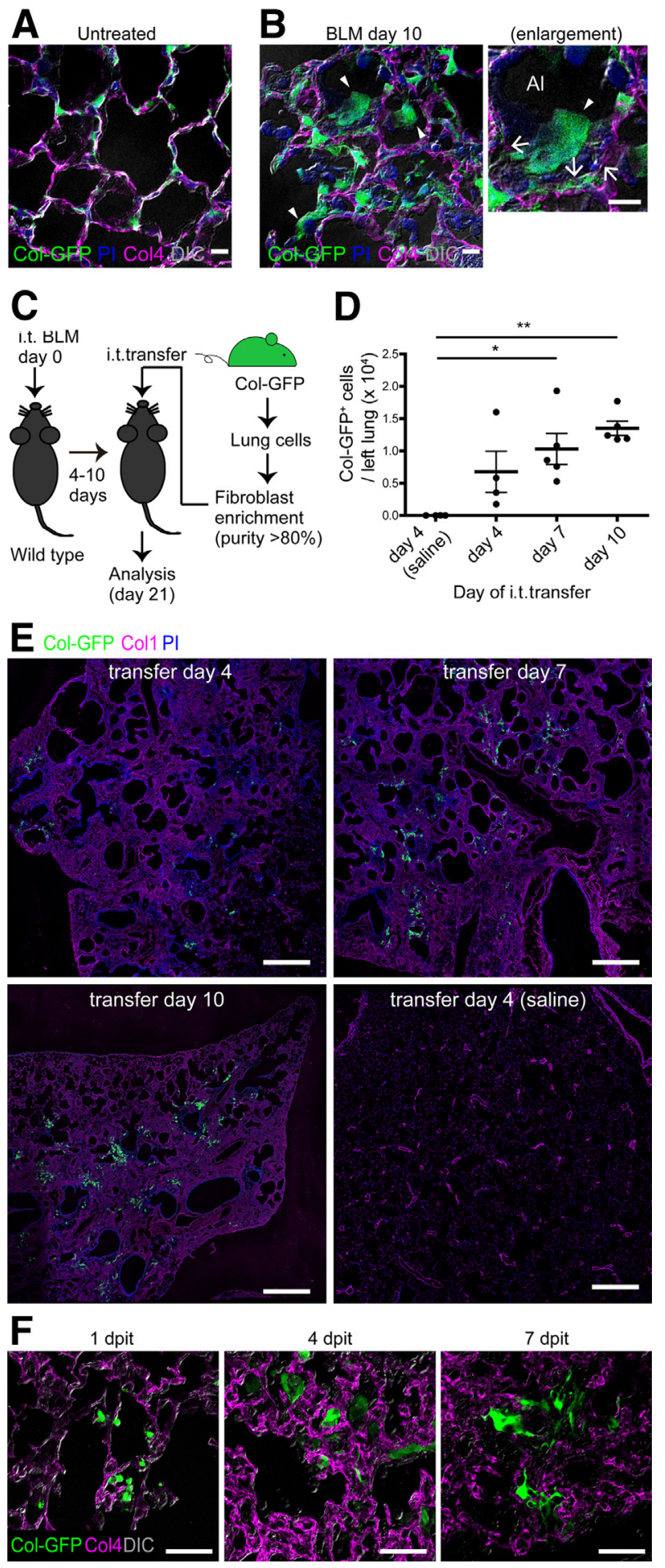

we next compared the potential of different lung cell subsets to contribute to the Col-GFP ${ }^{+}$profibrotic population. We purified Col-GFP ${ }^{+}, \mathrm{Col}_{-G F P^{+}} \mathrm{NG}^{-\mathrm{DsRed}^{+}, \mathrm{NG} \text {-DsRed }}{ }^{+}$, and EpCAM $^{+}$cells by fluorescence-activated cell sorting and performed intratracheal transfer $\left(5 \times 10^{4}\right.$ cells/mouse $) 7$ days after BLM treatment (Figure 3A). Col-GFP ${ }^{+}$cells were purified further by gating on autofluorescence ${ }^{+}$cells to exclude smooth muscle cells. After sorting, the purity was greater than 95\% (Figure 3B). On day 14 after BLM treatment, we counted the number of Col-GFP ${ }^{+}$cells in the lungs of host mice (Figure 3C and Supplemental Figure S3A). Although we detected Col-GFP ${ }^{+}$cells in the lungs of mice receiving Col$\mathrm{GFP}^{+}$NG2-DsRed ${ }^{+}$cell transfer $\left(8.11 \pm 2.44 \times 10^{2}\right.$ cells from the left lung), the number of cells detected was approximately $27 \%$ of that counted in mice receiving Col-GFP ${ }^{+}$cell transfer $\left(3.02 \pm 0.42 \times 10^{3}\right.$ cells from the left lung) (Figure 3C). Some of the transferred cells in mice receiving Col-GFP $^{+}$NG2-DsRed $^{+}$cell transfer lost NG2-DsRed expression (Supplemental Figure S3B). No Col-GFP ${ }^{+}$cells were detected in mice receiving NG2-DsRed ${ }^{+}$or $\mathrm{EpCAM}^{+}$ cell transfer (Figure 3C), whereas a small number of NG2DsRed $^{+}$cells without Col-GFP expression were detected in mice receiving NG2-DsRed ${ }^{+}$cell transfer $\left(5.40 \pm 0.68 \times 10^{2}\right.$ cells from the left lung) (Supplemental Figure S3C).

Histologic examination showed that dilated or stellate Col-GFP ${ }^{+}$cells formed fibroblastic foci in mice receiving $\mathrm{Col}_{-\mathrm{GFP}^{+}}$cell transfer (Figure 3D). In mice receiving Col$\mathrm{GFP}^{+}$NG2-DsRed ${ }^{+}$cell transfer, the transferred cells were incorporated sparsely into the interstitium and did not show enlarged morphology or cluster formation (Figure 3D). Transferred NG2-DsRed ${ }^{+}$cells also were incorporated into fibrotic areas, but their morphology of relatively small cell bodies and long processes was distinct from that of activated

\footnotetext{
Figure 2 Intratracheal transfer facilitates the engraftment of Col-green fluorescent protein (GFP) ${ }^{+}$cells in bleomycin (BLM)-treated lungs. Lung sections from untreated (A) or BLM-treated (B) mice (day 10). Col-GFP (green) mice were stained with PI (blue) and antibodies against Col4 (magenta). Arrowheads indicate Col-GFP ${ }^{+}$cells that migrated into alveolar airspaces. Arrows indicate the basement membrane of the alveolar walls. C: Design of intratracheal transfer experiments. Wild-type mice were treated with BLM before intratracheal administration of cell suspension. Col-GFP ${ }^{+}$resident fibroblasts were enriched by negative selection of CD31 CD45 ${ }^{-}$CD146- EpCAM $^{-}$Ter119- cells by magnetic-activated cell sorting (MACS). The purity of Col-GFP ${ }^{+}$platelet derived growth factor receptor (PDGFR) $\alpha^{+}$cells was greater than $80 \%$ in each experiment. D: Col-GFP cells $\left(1 \times 10^{6}\right)$ were transferred into BLM or saline-treated wild-type mice on day 4,7 , or 10 after BLM treatment. Twenty-one days after BLM or saline treatment, the number of Col-GFP ${ }^{+}$cells in the left lung was quantified by flow cytometry. Data are expressed as means \pm SEM and individual data points. E: Lung sections from host mice (BLM or saline day 21) were stained with PI and an antibody against type 1 collagen (Col1). F: Col-GFP ${ }^{+}$cells $\left(4 \times 10^{6}\right)$ were transferred into wild-type mice 7 days after BLM treatment. Lung sections from the mice 1, 4, and 7 days after intratracheal transfer (dpit) were stained for Col4. At least three independent experiments were performed for each analysis. $n=4$ to 5 (D). ${ }^{*} P<0.05,{ }^{*} P<<0.01$, oneway analysis of variance followed by the Tukey-Kramer post-test. Scale bars: $10 \mu \mathrm{m}$ (A and B), $500 \mu \mathrm{m}(\mathbf{E}), 50 \mu \mathrm{m}(\mathbf{F})$. Al, alveolar airspace; PI, propidium iodide.
} 

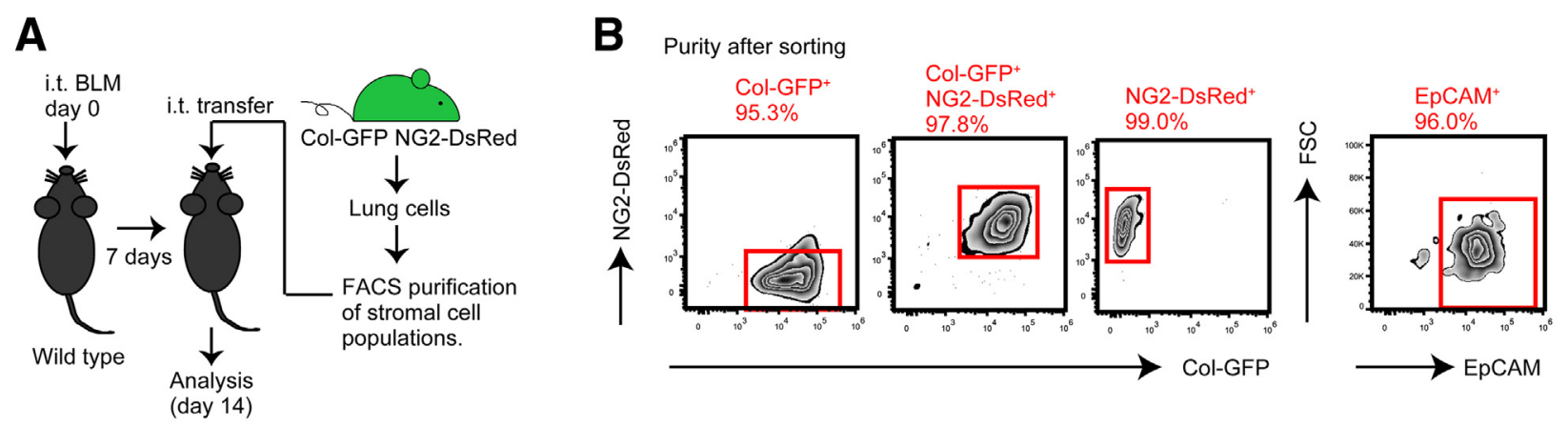

C
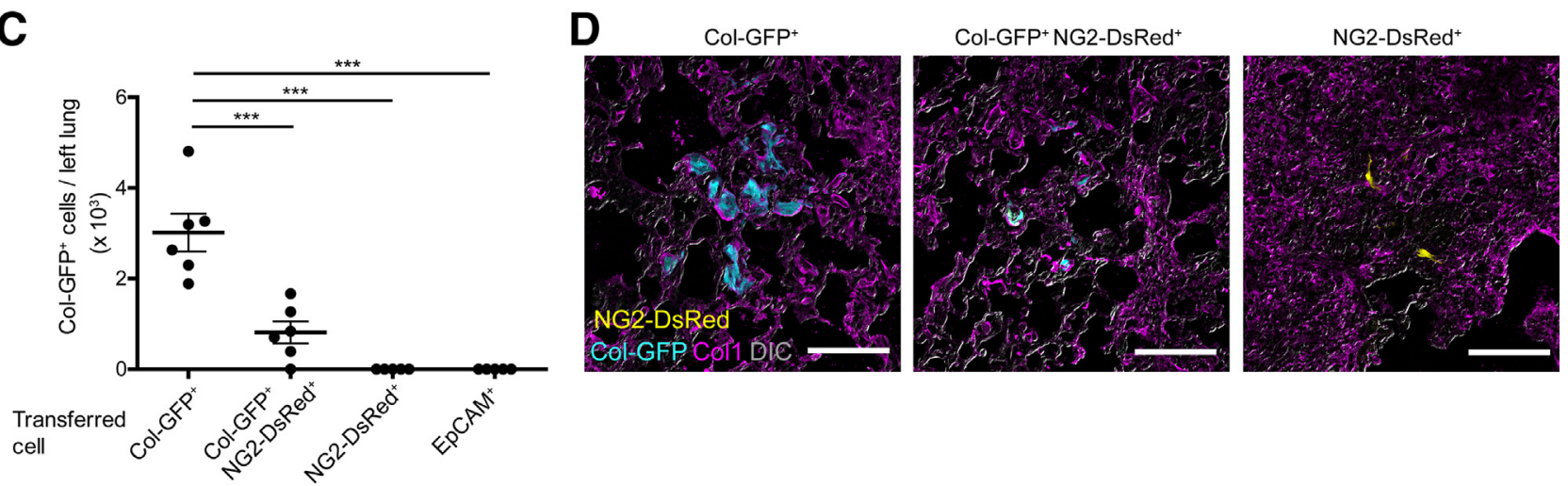

Figure 3 Lineage tracing of lung cell subsets by the intratracheal transfer approach. A: Experimental design. Each lung cell population was purified by fluorescence-activated cell sorting (FACS) and intratracheal transfer was performed ( $5 \times 10^{4}$ cells per mouse) into wild-type mice 7 days after bleomycin (BLM) treatment, followed by analysis another 7 days later. B: After sorting, purity was assessed by flow cytometry. Each cell population showed $>95 \%$ purity. C: 0 n day 14 after BLM treatment, the number of Col-GFP ${ }^{+}$cells in the left lungs of host mice were quantified by flow cytometry. Data are expressed as means \pm SEM and individual data points. D: Lung sections from host mice on day 14 after BLM treatment were stained for Col1. $n=5$ to 6 (C). ${ }^{* * * P<0.001, ~ o n e-w a y ~}$ analysis of variance followed by the Tukey-Kramer post-test. Scale bars: $100 \mu \mathrm{m}$. Two independent experiments were performed for each analysis.

fibroblasts (Figure 3D). Because of the absence of fibroblastic foci formed by Col-GFP ${ }^{+}$cells in mice receiving Col-GFP ${ }^{+}$ NG2-DsRed ${ }^{+}$cell transfer, engraftment of Col-GFP ${ }^{+}$NG2$\operatorname{DsRed}^{+}$cells may have resulted from ectopic incorporation of perivascular smooth muscle cells. Our results confirm that $\mathrm{EpCAM}^{+}$cells can be engrafted as epithelial cells (Supplemental Figure S3D), as reported previously, ${ }^{24,25}$ suggesting that these cells did not differentiate into Col$\mathrm{GFP}^{+}$cells through epithelial-to-mesenchymal transition. Taken together with the abundance of Col-GFP ${ }^{+}$cells in the lungs (Figure 1A), these results strongly suggest that resident fibroblasts are the most profibrotic cell population in the lungs, and that neither NG2-DsRed ${ }^{+}$pericytes nor $\mathrm{EpCAM}^{+}$epithelial cells contribute to the Col-GFP ${ }^{+}$ profibrotic cell population when administered by intratracheal transfer.

We further investigated the activation of resident fibroblasts in the intratracheal transfer model by measuring gene expression and performing BrdU labeling. Transferred Col$\mathrm{GFP}^{+}$cells dramatically up-regulated profibrotic genes such as Collal, Acta2, Spp 1, S100a4, Fn1, and Tnc up to 7 dpit (BLM day 14) (Figure 4A). ${ }^{6}$ Col-GFP ${ }^{+}$cells in some fibroblastic foci highly expressed $\alpha$-SMA (Figure 4B), with a pronounced increase in $\alpha$-SMA expression occurring between 2 and 4 dpit (Figure 4C and Supplemental Figure S4A). To investigate the proliferation of Col-GFP ${ }^{+}$cells, we transferred cells into host mice that were treated with drinking water containing BrdU beginning 1 day before intratracheal transfer and continuing until analysis. In these experiments, $3.93 \% \pm 0.63 \%$ of Col$\mathrm{GFP}^{+}$cells were $\mathrm{BrdU}^{+}$at $2 \mathrm{dpit}$, increasing to $7.70 \% \pm 0.40 \%$ at 4 dpit (Figure 4D and Supplemental Figure S4B). The proportion of $\mathrm{BrdU}^{+}$Col-GFP ${ }^{+}$cells did not increase between 4 and 7 dpit, suggesting that the proliferation of Col-GFP ${ }^{+}$cells peaked at approximately 4 dpit (Figure 4D). Some, but not all, Col-GFP ${ }^{+}$cells in fibroblastic foci were $\mathrm{BrdU}^{+}$(Figure 4E). The fibroblastic foci formed by transferred fibroblasts were localized outside the basement membrane and directly covered by epithelium, an anatomic characteristic similar to that observed in IPF patients (Figure 4E) ${ }^{23,26-28}$ Transferred cells also dramatically increased in size and intracellular complexity between 2 and $4 \mathrm{dpit}$, after which these parameters decreased slightly up to 7 dpit (Supplemental Figure S4, C and D). Transferred Col-GFP ${ }^{+}$cells that were purified at 4 dpit showed increased migratory capacity (Figure $4 \mathrm{~F}$ and Supplemental Figure S4E) and increased invasive capacity (Supplemental Figure S4F). These results suggest that resident fibroblasts exposed to the fibrotic environment proliferate and acquire a profibrotic signature within 4 days, after which they enter another phase where they are less proliferative but continue to express profibrotic genes.

Finally, we examined whether the increased number of activated fibroblasts present resulting from the transfer of resident fibroblasts correlates with increased total collagen deposition. The number of engrafted Col-GFP ${ }^{+}$cells 

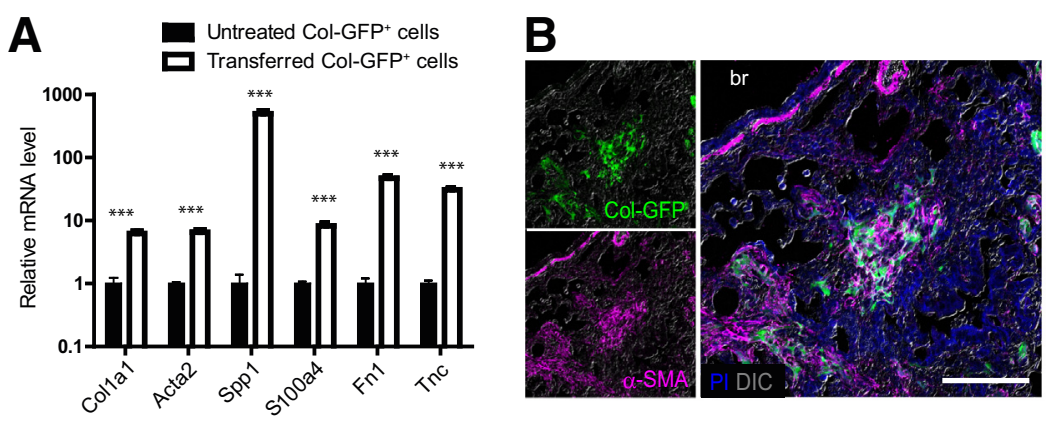

C
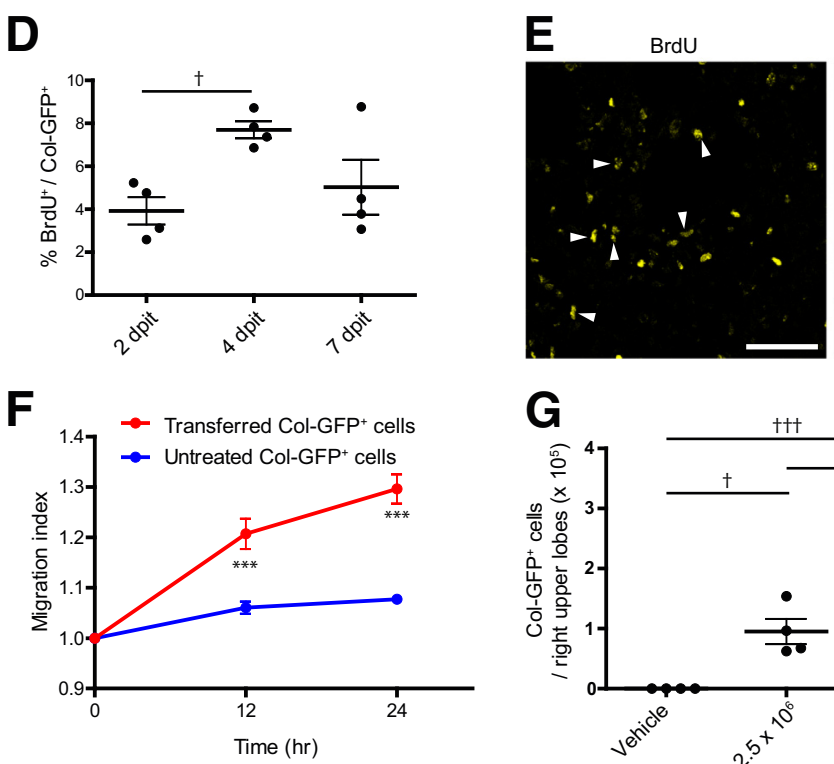

G

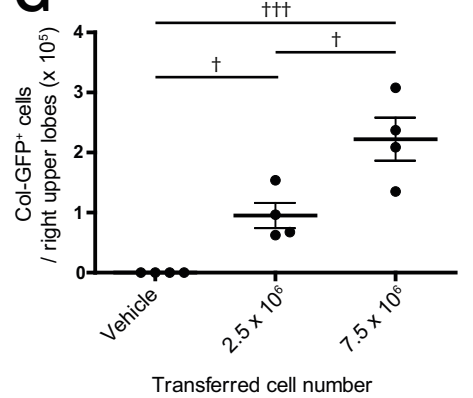

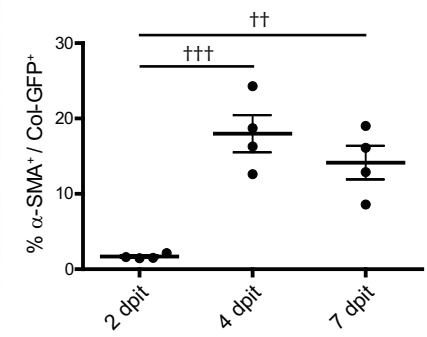
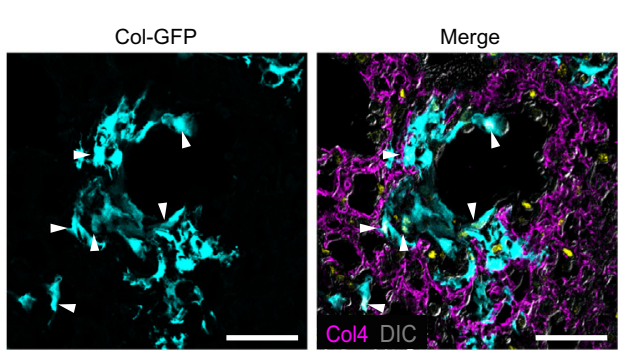

H

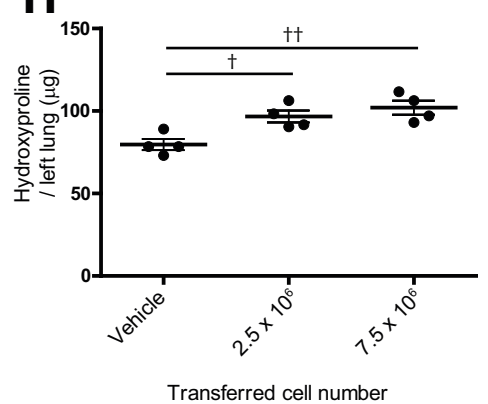

Figure 4 Intratracheal transfer shows profibrotic activation of Col-green fluorescent protein (GFP) ${ }^{+}$cells. Intratracheal transfer was performed with magnetic-

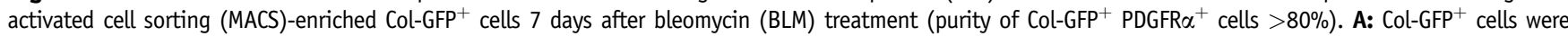
transferred into wild-type mice. At $7 \mathrm{dpit}$, Col-GFP ${ }^{+}$cells were purified by fluorescence-activated cell sorting (FACS) for mRNA extraction and measurement of profibrotic gene expression by quantitative real-time PCR. B: Lung sections from host mice at 7 days after intratracheal transfer (dpit) were stained with propidium iodide (PI) (blue) and an antibody against $\alpha$-smooth muscle actin ( $\alpha$-SMA) (magenta). Col-GFP (green). C: Intratracheal transfer was performed on wild-type mice and Col-GFP ${ }^{+}$ cells were analyzed for $\alpha$-SMA expression at 2, 4, and 7 dpit by flow cytometry. D and E: Bromodeoxyuridine (BrdU) was included in the drinking water of wild-type mice commencing 1 day before intratracheal transfer and continuing until analysis. D: BrdU incorporation was analyzed at 2, 4, and 7 dpit by flow cytometry. E: Lung sections from host mice at 4 dpit were stained for BrdU (yellow) and Col4 (magenta). Arrowheads indicate BrdU Col-GFP $^{+}$cells (cyan). F: The migratory capacity of FACS-purified Col-GFP ${ }^{+}$cells at 4 dpit and untreated Col-GFP ${ }^{+}$cells was assessed using a gap migration assay. Col-GFP ${ }^{+}$cells were purified from pooled whole-lung cells from five host mice or three untreated Col-GFP mice. $\mathbf{G}$ and $\mathbf{H}$ : Intratracheal transfer of 2.5 or $7.5 \times 10^{6} \mathrm{Col}_{-G_{F P}}{ }^{+}$cells was performed on wild-type mice, with phosphate-buffered saline (PBS) instilled for the vehicle group. The lungs of host mice were analyzed at $7 \mathrm{dpit}$. G: Col-GFP ${ }^{+}$cells in the apical and azygous lobes of the lung were counted by flow cytometry. $\mathbf{H}$ : The hydroxyproline content in the left lung was measured. Data are expressed as means \pm SEM $(\mathbf{A})$, means of three technical replicates \pm SEM $(\mathbf{F})$, or means \pm SEM and individual data points (C, D, G, and $\mathbf{H})$. At least two independent experiments were performed for each analysis. $n=3\left(\mathbf{A}\right.$, untreated Col-GFP ${ }^{+}$cells; $n=5$ (A, transferred Col-GFP ${ }^{+}$cells); $n=4$ per group $(\mathbf{C}-\mathbf{E}, \mathbf{G}$, and $\mathbf{H}) .{ }^{* *} P<0.001$, Student's $t$-test compared with the untreated group; ${ }^{\dagger} P<0.05,{ }^{\dagger \dagger} P<0.01$, and ${ }^{\dagger \dagger} P<0.001$, one-way analysis of variance followed by the Tukey-Kramer post-test. Scale bars: $100 \mu \mathrm{m}$ (B); $50 \mu \mathrm{m}(\mathbf{E})$. br, bronchiole.

increased in a dose-dependent manner (Figure 4G). The amount of collagen in the lungs significantly increased in mice receiving intratracheal transfer (Figure $4 \mathrm{H}$ ). On the other hand, intratracheal transfer did not augment inflammatory cell infiltration into the lungs compared with vehicletreated mice (Supplemental Figure S5A). The mRNA expression of previously reported profibrotic cytokines in whole-lung cells also suggested that the intratracheal transfer procedure did not enhance the expression of profibrotic cytokines (Supplemental Figure S5B). ${ }^{29}$ These data suggest that the increase of collagen deposition observed in mice receiving intratracheal transfer was caused by the increased number of activated fibroblasts in the lungs of these mice, not by augmented profibrotic responses in the host cells. A negative feedback mechanism has been reported to regulate collagen deposition, ${ }^{30}$ which may explain why there was no significant difference in the amount of collagen observed in the low- and high-dose groups (Figure $4 \mathrm{H}$ ).

\section{Discussion}

Understanding the cellular origin of activated fibroblasts may lead to novel therapeutic approaches that suppress the 
progression of irreversible tissue remodeling in fibrotic diseases. In the present study we have shown that intratracheal transfer of resident fibroblasts recapitulates part of the fibrotic process, however, neither pericytes labeled with NG2-DsRed nor $\mathrm{EpCAM}^{+}$epithelial cells differentiate into profibrotic cells after intratracheal transfer. We also have provided in vivo evidence that fibroblasts in alveolar airspaces form fibroblastic foci with similar characteristics to those seen in IPF patients.

The migration of fibroblasts to sites of injury is a natural part of the wound healing process. In dermal injury, fibroblasts migrate into a provisional matrix formed in fibrin clots, where they differentiate into myofibroblasts, enabling contraction. ${ }^{31} \mathrm{~A}$ similar wound healing process is suggested to occur in lung injury. ${ }^{20,21}$ Fibroblasts isolated from the lungs of IPF patients show enhanced migratory potential. ${ }^{32}$ In addition, previous reports have suggested, based on anatomic characteristics, that fibroblastic foci in IPF patients are formed by the migration of fibroblasts into the alveolar airspaces. ${ }^{23,26}$ Our histologic analyses using Col-GFP mice confirm the migration of activated fibroblasts into alveolar airspaces after lung injury, as reported previously. Because intratracheal transfer delivered resident fibroblasts directly to the sites of injury that endogenous fibroblasts migrated into, it is not surprising that transferred fibroblasts showed similar activation signatures to endogenous fibroblasts. At the same time, the profibrotic activation and fibroblastic foci formation observed after intratracheal transfer strongly suggest that exposure to alveolar airspaces plays an important role in the fibrotic process for resident fibroblasts.

Resident fibroblasts did not engraft into uninjured lungs, indicating that an inflammatory environment and loss of epithelial integrity are necessary for the survival and activation of resident fibroblasts in the alveolar airspaces. This result also indicates that purifying engrafted cells after intratracheal transfer concentrates activated fibroblasts, whereas endogenous whole-lung cells after bleomycin treatment are a mixture of activated fibroblasts from fibrotic lesions and normal fibroblasts from uninjured areas. Tager et $\mathrm{al}^{33}$ showed that the inhibition of fibroblast chemoattractant in the alveolar airspaces can be an effective therapeutic strategy. In addition, $\mathrm{Li}$ et $\mathrm{al}^{34}$ showed that enhancing the invasive phenotype of myofibroblasts exacerbates pulmonary fibrosis. Because our intratracheal transfer approach provides various options for investigating the molecular mechanisms of fibroblast activation in the alveolar airspaces, further exploration of the key molecules that modulate activation signatures by this approach has the potential to show novel therapeutic targets.

By using the intratracheal transfer method, we showed that NG2-DsRed ${ }^{+}$cells were pericytes in the alveolar walls and these cells did not have the potential to become profibrotic cells. This finding is inconsistent with the findings reported by Hung et $\mathrm{al},{ }^{7}$ which suggested a major contribution of pericytes to the myofibroblast population. The main difference between the present study and the study by Hung et $\mathrm{al}^{7}$ is that we transferred normal pericytes directly into the inflammatory environment in the alveolar airspaces. However, our results do not exclude the possibility that pericytes contribute to the profibrotic cell population when they are educated in a perivascular niche and thereby become activated. Another possible reason for the discrepancy between our results and those of Hung et al ${ }^{7}$ is that NG2-DsRed labels only a portion of PDGFR $\beta^{+}$cells in the alveolar walls. Although Hung et al ${ }^{7}$ showed that approximately $60 \%$ of Foxd1-Cre-labeled cells express NG2, it is possible that $\mathrm{NG}^{-} \mathrm{PDGFR}^{+}$cells are myofibroblast progenitors. This hypothesis also may explain the results of Rock et al, ${ }^{5}$ who reported a minor contribution of NG2-CreER-labeled cells to the myofibroblast population. Thus, the heterogeneity and the profibrotic potential of the PDGFR $\beta^{+}$cell population require further investigation.

In this study, we examined the fate of resident fibroblasts in pulmonary fibrosis using an intratracheal transfer approach that partly recapitulated the profibrotic process undergone by endogenous fibroblasts. Our results confirm the activation and key role in collagen deposition of resident fibroblasts. This study not only presents the results of rigorous lineage tracing of resident fibroblasts, but also highlights the advantages of the intratracheal transfer approach for investigating the profibrotic actions of fibroblasts in pulmonary fibrosis.

\section{Acknowledgments}

We thank Shun-ichi Fujita, Shin Aoki, Ai Yamashita, and other colleagues in the laboratory for their assistance with the experiments presented in this study.

T.T., S.U., and K.M. designed the study and analyzed and interpreted the data; T.T. performed the experiments; Y.I. provided Col-GFP mice; T.T., S.U., S.S., and K.M. wrote the manuscript; and K.M. supervised the study.

\section{Supplemental Data}

Supplemental material for this article can be found at http://dx.doi.org/10.1016/j.ajpath.2015.07.022.

\section{References}

1. Noble PW, Barkauskas CE, Jiang D: Pulmonary fibrosis: patterns and perpetrators. J Clin Invest 2012, 122:2756-2762

2. Barkauskas CE, Noble PW: Cellular mechanisms of tissue fibrosis. 7. New insights into the cellular mechanisms of pulmonary fibrosis. Am J Physiol Cell Physiol 2014, 306:C987-C996

3. King TE Jr, Pardo A, Selman M: Idiopathic pulmonary fibrosis. Lancet 2011, 378:1949-1961

4. Kramann R, DiRocco DP, Humphreys BD: Understanding the origin, activation and regulation of matrix-producing myofibroblasts for treatment of fibrotic disease. J Pathol 2013, 231:273-289

5. Rock JR, Barkauskas CE, Cronce MJ, Xue Y, Harris JR, Liang J, Noble PW, Hogan BLM: Multiple stromal populations contribute to pulmonary fibrosis without evidence for epithelial to mesenchymal transition. Proc Natl Acad Sci U S A 2011, 108:E1475-E1483

6. Tsukui T, Ueha S, Abe J, Hashimoto S, Shichino S, Shimaoka T, Shand FH, Arakawa Y, Oshima K, Hattori M, Inagaki Y, Tomura M, Matsushima K: Qualitative rather than quantitative changes are 
hallmarks of fibroblasts in bleomycin-induced pulmonary fibrosis. Am J Pathol 2013, 183:758-773

7. Hung C, Linn G, Chow YH, Kobayashi A, Mittelsteadt K, Altemeier WA, Gharib SA, Schnapp LM, Duffield JS: Role of lung pericytes and resident fibroblasts in the pathogenesis of pulmonary fibrosis. Am J Respir Crit Care Med 2013, 188:820-830

8. Goritz C, Dias DO, Tomilin N, Barbacid M, Shupliakov O, Frisen J: A pericyte origin of spinal cord scar tissue. Science 2011, 333:238-242

9. Dulauroy S, Di Carlo SE, Langa F, Eberl G, Peduto L: Lineage tracing and genetic ablation of ADAM12(+) perivascular cells identify a major source of profibrotic cells during acute tissue injury. Nat Med 2012, 18:1262-1270

10. Kramann R, Schneider RK, DiRocco DP, Machado F, Fleig S, Bondzie PA, Henderson JM, Ebert BL, Humphreys BD: Perivascular Gli1+ progenitors are key contributors to injury-induced organ fibrosis. Cell Stem Cell 2015, 16:51-66

11. Higashiyama R, Moro T, Nakao S, Mikami K, Fukumitsu H, Ueda Y, Ikeda K, Adachi E, Bou-Gharios G, Okazaki I, Inagaki Y: Negligible contribution of bone marrow-derived cells to collagen production during hepatic fibrogenesis in mice. Gastroenterology 2009, 137: 1459-1466.e1

12. Tomura M, Mori YS, Watanabe R, Tanaka M, Miyawaki A, Kanagawa O: Time-lapse observation of cellular function with fluorescent probe reveals novel CTL-target cell interactions. Int Immunol 2009, 21:1145-1150

13. Hogan BL, Barkauskas CE, Chapman HA, Epstein JA, Jain R, Hsia CC, Niklason L, Calle E, Le A, Randell SH, Rock J, Snitow M, Krummel M, Stripp BR, Vu T, White ES, Whitsett JA, Morrisey EE: Repair and regeneration of the respiratory system: complexity, plasticity, and mechanisms of lung stem cell function. Cell Stem Cell 2014, 15:123-138

14. Song S, Ewald AJ, Stallcup W, Werb Z, Bergers G: PDGFRbeta+ perivascular progenitor cells in tumours regulate pericyte differentiation and vascular survival. Nat Cell Biol 2005, 7:870-879

15. Middleton J, Americh L, Gayon R, Julien D, Mansat M, Mansat P, Anract P, Cantagrel A, Cattan P, Reimund JM, Aguilar L, Amalric F, Girard JP: A comparative study of endothelial cell markers expressed in chronically inflamed human tissues: MECA-79, Duffy antigen receptor for chemokines, von Willebrand factor, CD31, CD34, CD105 and CD146. J Pathol 2005, 206:260-268

16. Armulik A, Genové G, Betsholtz C: Pericytes: developmental, physiological, and pathological perspectives, problems, and promises. Dev Cell 2011, 21:193-215

17. Barkauskas CE, Cronce MJ, Rackley CR, Bowie EJ, Keene DR, Stripp BR, Randell SH, Noble PW, Hogan BL: Type 2 alveolar cells are stem cells in adult lung. J Clin Invest 2013, 123:3025-3036

18. Kisseleva T, Cong M, Paik Y, Scholten D, Jiang C, Benner C, Iwaisako K, Moore-Morris T, Scott B, Tsukamoto H, Evans SM, Dillmann W, Glass CK, Brenner DA: Myofibroblasts revert to an inactive phenotype during regression of liver fibrosis. Proc Natl Acad Sci U S A 2012, 109:9448-9453

19. Sirianni FE, Chu FS, Walker DC: Human alveolar wall fibroblasts directly link epithelial type 2 cells to capillary endothelium. Am J Respir Crit Care Med 2003, 168:1532-1537

20. Fukuda Y, Ferrans VJ, Schoenberger CI, Rennard SI, Crystal RG: Patterns of pulmonary structural remodeling after experimental paraquat toxicity. The morphogenesis of intraalveolar fibrosis. Am J Pathol 1985, 118:452-475

21. Fukuda Y, Basset F, Ferrans VJ, Yamanaka N: Significance of early intra-alveolar fibrotic lesions and integrin expression in lung biopsy specimens from patients with idiopathic pulmonary fibrosis. Hum Pathol 1995, 26:53-61

22. Noble PW: Back to the future: historical perspective on the pathogenesis of idiopathic pulmonary fibrosis. Am J Respir Cell Mol Biol 2005, 33:113-120

23. Kuhn C 3rd, Boldt J, King TE Jr, Crouch E, Vartio T, McDonald JA: An immunohistochemical study of architectural remodeling and connective tissue synthesis in pulmonary fibrosis. Am Rev Respir Dis 1989, 140:1693-1703

24. Vaughan AE, Brumwell AN, Xi Y, Gotts JE, Brownfield DG, Treutlein B, Tan K, Tan V, Liu FC, Looney MR, Matthay MA, Rock JR, Chapman HA: Lineage-negative progenitors mobilize to regenerate lung epithelium after major injury. Nature 2015, 517: $621-625$

25. Zuo W, Zhang T, Wu DZ, Guan SP, Liew AA, Yamamoto Y, Wang X, Lim SJ, Vincent M, Lessard M, Crum CP, Xian W, McKeon F: p63(+) $\mathrm{Krt5}(+)$ distal airway stem cells are essential for lung regeneration. Nature 2015, 517:616-620

26. Kuhn C, McDonald JA: The roles of the myofibroblast in idiopathic pulmonary fibrosis. Ultrastructural and immunohistochemical features of sites of active extracellular matrix synthesis. Am J Pathol 1991, 138: $1257-1265$

27. Brody AR, Craighead JE: Interstitial associations of cells lining air spaces in human pulmonary fibrosis. Virchows Arch A Pathol Anat Histol 1976, 372:39-49

28. Brody AR, Soler P, Basset F, Haschek WM, Witschi H: Epithelialmesenchymal associations of cells in human pulmonary fibrosis and in BHT-oxygen-induced fibrosis in mice. Exp Lung Res 1981, 2: 207-220

29. Wynn TA: Integrating mechanisms of pulmonary fibrosis. J Exp Med 2011, 208:1339-1350

30. Xia H, Nho R, Kleidon J, Kahm J, Henke CA: Polymerized collagen inhibits fibroblast proliferation via a mechanism involving the formation of a 1 integrin-protein phosphatase 2A-tuberous sclerosis complex 2 complex that suppresses S6K1 activity. J Biol Chem 2008, 283: 20350-20360

31. Tomasek JJ, Gabbiani G, Hinz B, Chaponnier C, Brown RA: Myofibroblasts and mechano-regulation of connective tissue remodelling. Nat Rev Mol Cell Biol 2002, 3:349-363

32. Suganuma H, Sato A, Tamura R, Chida K: Enhanced migration of fibroblasts derived from lungs with fibrotic lesions. Thorax 1995, 50: 984-989

33. Tager AM, LaCamera P, Shea BS, Campanella GS, Selman M, Zhao Z, Polosukhin V, Wain J, Karimi-Shah BA, Kim ND, Hart WK, Pardo A, Blackwell TS, Xu Y, Chun J, Luster AD: The lysophosphatidic acid receptor LPA1 links pulmonary fibrosis to lung injury by mediating fibroblast recruitment and vascular leak. Nat Med 2008, 14: $45-54$

34. Li Y, Jiang D, Liang J, Meltzer EB, Gray A, Miura R, Wogensen L, Yamaguchi Y, Noble PW: Severe lung fibrosis requires an invasive fibroblast phenotype regulated by hyaluronan and CD44. J Exp Med 2011, 208:1459-1471 